Institut für Mathematik

\title{
Multiple Shooting for Unstructured \\ Nonlinear Differential-Algebraic Equations of Arbitrary Index
}

\author{
P. Kunkel, V. Mehrmann and R. Stöver
}

Technical Report 751-02

Preprint-Reihe des Instituts für Mathematik

Technische Universität Berlin 



\title{
Multiple Shooting for Unstructured Nonlinear Differential-Algebraic Equations of Arbitrary Index
}

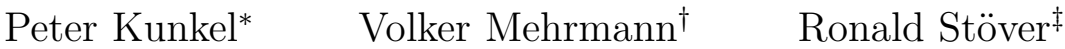

December 2, 2002

\begin{abstract}
We study multiple shooting methods for the numerical solution of nonlinear boundary value problems for unstructured nonlinear systems of differential-algebraic equations with arbitrary index. We give a concergence analysis and demonstrate the results with some numerical examples.
\end{abstract}

\section{Introduction}

In this paper we consider the numerical solution of nonlinear boundary value problems for systems of differential-algebraic equations of arbitrary index. Of the many possible solution approaches we discuss in this paper how to develop multiple shooting methods for these boundary value problems. Multiple shooting is well studied and widely used for ordinary differential equations, see [1], and also for special classes of systems of differential-algebraic equations (DAEs), see [15, 18].

In this paper we study general nonlinear DAE boundary value problems, i. e., problems of the form

$$
\begin{array}{ll}
\text { (a) } & F(t, x, \dot{x})=0, \\
\text { (b) } & r(x(\underline{t}), x(\bar{t}))=0,
\end{array}
$$

where $F:[\underline{t}, \bar{t}] \times \mathbb{D}_{x} \times \mathbb{D}_{\dot{x}} \rightarrow \mathbb{R}^{n}, r: \mathbb{D}_{x} \times \mathbb{D}_{x} \rightarrow \mathbb{R}^{d}$ with $\mathbb{D}_{x}, \mathbb{D}_{\dot{x}} \subseteq \mathbb{R}^{n}$ open. The integer $d$ denotes the number of differential components of $x$. A precise definition will follow in the next section.

The typical feature of shooting methods is that the solution of (1.1) is achieved through the solution of initial value problems, where it is implicitly assumed that these are wellconditioned and can be solved sufficiently accurate. The boundary condition, together with the continuity conditions in the case of multiple shooting, then form a system of nonlinear equations for the initial values. In contrast to the application of shooting methods for the solution of ordinary differential equations, however, a problem arises for DAEs due to the fact that initial values have to be consistent with all explicit and hidden algebraic constraints. But

\footnotetext{
${ }^{*}$ Mathematisches Institut, Universität Leipzig, Augustusplatz 10-11, D-04109 Leipzig, Fed. Rep. Germany. Supported by DFG research grant Ku964/4.

${ }^{\dagger}$ Institut für Mathematik, MA 4-5, Technische Universität Berlin, Straße des 17. Juni 136, D-10623 Berlin, Fed. Rep. Germany. Supported by DFG research grant Me790/11.

${ }^{\ddagger}$ Fachbereich 3 - Mathematik und Informatik, Universität Bremen, Postfach 330 440, D-28334 Bremen, Fed. Rep. Germany.
} 
even starting with a consistent initial guess, the iterative solver for the nonlinear equation will in general produce corrections that lead to inconsistent intermediate iterates. For this reason, shooting methods for nonlinear DAE boundary value problems were only considered in very special cases, where the algebraic constraints are known explicitly [18], or where they can be accessed due to the special structure of the equation $[5,15]$.

For general linear problems with variable coefficients, the set of consistent initial values at a given point forms an affine space which is numerically accessible, see [9, 12]. Based on this knowledge shooting methods were developed for this case in [20]. To generalize this approach to the general nonlinear case is the subject of the present paper. The new method that we present is able to treat boundary value problems for general DAEs of a given arbitrary index, i. e., there are no assumptions on the structure of the equations besides the requirement that the DAE can be assigned a certain kind of index. Note that such an assumption is indispensable because we need existence and uniqueness results on which we can base our method.

The paper is organized as follows. In Section 2, we state some preliminaries on the theory of DAEs. In particular, we give the basic index definition and some further results that we need for the construction and investigation of the presented approach. In Section 3 we discuss the local uniqueness of solutions of (1.1) via single shooting. A multiple shooting approach is then presented in Section 4 together with a special Gauß-Newton-like method. In particular, we show that the arising linear equations can be reduced to shooting systems as they are obtained by multiple shooting for a system of $d$ ordinary differential equations. In Section 5 , we then discuss the results of a number of numerical experiments. Finally, we give some conclusions in Section 6.

\section{Preliminaries}

In order to analyze differential-algebraic equations, we first need the notion of index. Loosely speaking, the index is the number of differentiations that we must apply to (1.1a) to obtain all algebraic constraints that (1.1a) imposes on possible values for $x(t)$. Assuming in the following that all occuring functions are sufficiently smooth, we first introduce the so-called derivative array functions (see $[2,3])$

$$
F_{\ell}\left(t, x, \dot{x}, \ldots, x^{(\ell+1)}\right)=\left[\begin{array}{c}
F(t, x, \dot{x}) \\
\frac{d}{d t} F(t, x, \dot{x}) \\
\vdots \\
\left(\frac{d}{d t}\right)^{\ell} F(t, x, \dot{x})
\end{array}\right]
$$

that are obtained from (1.1a) by successive differentiation with respect to $t$. Note that we treat $\left(t, x, \dot{x}, \ldots, x^{(\ell+1)}\right)$ here as independent variables such that $F_{\ell}$ is a function from some subset of $\mathbb{R}^{(\ell+2) n+1}$ into $\mathbb{R}^{(\ell+1) n}$. Partial derivatives will be denoted by corresponding subscripts as, e. g., in

$$
F_{\ell ; x}=\frac{\partial}{\partial x} F_{\ell}, \quad F_{\ell ; \dot{x}, \ldots, x^{(\ell+1)}}=\left[\begin{array}{lll}
\frac{\partial}{\partial \dot{x}} F_{\ell} & \cdots & \frac{\partial}{\partial x^{(\ell+1)}} F_{\ell}
\end{array}\right] .
$$

The following hypothesis states the central requirements on the differential-algebraic equation (1.1a), see [10]. 
Hypothesis 2.1 There exist integers $\mu, a$ and $d$ such that for all values $\left(t, x, \dot{x}, \ldots, x^{(\mu+1)}\right) \in$ $\mathbb{L}_{\mu}$ with

$$
\mathbb{L}_{\mu}=\left\{\left(t, x, \dot{x}, \ldots, x^{(\mu+1)}\right) \in \mathbb{R}^{(\mu+2) n+1} \mid F_{\mu}\left(t, x, \dot{x}, \ldots, x^{(\mu+1)}\right)=0\right\} \neq \emptyset
$$

associated with $F$ the following properties hold:

1. We have

$$
\operatorname{rank} F_{\mu ; \dot{x}, \ldots, x^{(\mu+1)}}\left(t, x, \dot{x}, \ldots, x^{(\mu+1)}\right)=(\mu+1) n-a,
$$

such that there exists a smooth matrix function $\hat{Z}_{2}$ on $\mathbb{L}_{\mu}$ with orthonormal columns and size $((\mu+1) n, a)$ satisfying

$$
\hat{Z}_{2}^{T} F_{\mu ; \dot{x}, \ldots, x^{(\mu+1)}}=0 \quad \text { on } \mathbb{L}_{\mu} .
$$

2. We have

$$
\operatorname{rank} \hat{Z}_{2}^{T} F_{\mu ; x}\left(t, x, \dot{x}, \ldots, x^{(\mu+1)}\right)=a,
$$

such that there exists a smooth matrix function $\hat{T}_{2}$ on $\mathbb{L}_{\mu}$ with orthonormal columns and size $(n, d)$, where $d=n-a$, satisfying

$$
\hat{Z}_{2}^{T} F_{\mu ; x} \hat{T}_{2}=0 \quad \text { on } \mathbb{L}_{\mu} .
$$

3. We have

$$
\operatorname{rank} F_{\dot{x}} \hat{T}_{2}\left(t, x, \dot{x}, \ldots, x^{(\mu+1)}\right)=d,
$$

such that there exists a smooth matrix function $\hat{Z}_{1}$ on $\mathbb{L}_{\mu}$ with orthonormal columns and size $(n, d)$ satisfying

$$
\operatorname{rank} \hat{Z}_{1}^{T} F_{\dot{x}} \hat{T}_{2}=d \quad \text { on } \mathbb{L}_{\mu}
$$

The minimal number $\mu$ (if it exists) such that Hypothesis 2.1 is fulfilled is called the strangeness index of $F$. The numbers $a$ and $d$ denote the size of the algebraic and differential part of (1.1a). In particular, the choice of initial values is restricted by $a$ algebraic constraints. More specific, for an initial value problem consisting of (1.1a) together with $x\left(t_{0}\right)=x_{0}$ to be solvable, the initial value $x_{0}$ must be extendable to a point $\left(t_{0}, x_{0}, \dot{x}_{0}, \ldots, x_{0}^{(\mu+1)}\right)$ in $\mathbb{L}_{\mu}$. This requirement can be reduced to $a$ conditions on $x_{0}$ itself. See [10] for more details.

A typical ingredient in the investigation of numerical methods for boundary value problems is the assumption that a solution of the given problem does exist. We therefore assume that there exists a sufficiently smooth solution $x^{*} \in C^{1}\left([\underline{t}, \bar{t}], \mathbb{R}^{n}\right)$ of $(1.1)$ in the sense that

(a) $\quad F\left(t, x^{*}(t), \dot{x}^{*}(t)\right)=0 \quad$ for all $t \in[\underline{t}, \bar{t}]$,

(b) $\quad F_{\mu}\left(t, x^{*}(t), P(t)\right)=0$ for all $t \in[\underline{t}, \bar{t}]$,

(c) $\quad r\left(x^{*}(\underline{t}), x^{*}(\bar{t})\right)=0$,

where $P:[\underline{t}, \bar{t}] \rightarrow \mathbb{R}^{(\mu+1) n}$ is some smooth function that coincides with $\dot{x}^{*}$ in the first $n$ components. See [11, Theorem 3] for sufficient conditions for such a function to exist.

Restricting the projectors $\hat{Z}_{1}, \hat{Z}_{2}$ and $\hat{T}_{2}$ of Hypothesis 2.1 to the path $\left(t, x^{*}(t), P(t)\right)$ which lies in $\mathbb{L}_{\mu}$ due to $(2.3 \mathrm{~b})$, we obtain functions

$$
Z_{1}:[\underline{t}, \bar{t}] \rightarrow \mathbb{R}^{n, d}, \quad Z_{2}:[\underline{t}, \bar{t}] \rightarrow \mathbb{R}^{(\mu+1) n, a}, \quad T_{2}:[\underline{t}, \bar{t}] \rightarrow \mathbb{R}^{n, d}
$$


that satisfy

(a) $\quad Z_{2}(t)^{T} F_{\mu ; \dot{x}, \ldots, x(\mu+1)}\left(t, x^{*}(t), P(t)\right)=0 \quad$ for all $t \in[\underline{t}, \bar{t}]$,

(b) $\quad Z_{2}(t)^{T} F_{\mu ; x}\left(t, x^{*}(t), P(t)\right) T_{2}(t)=0 \quad$ for all $t \in[\underline{t}, \bar{t}]$,

(c) $\quad \operatorname{rank} Z_{1}(t)^{T} F_{\dot{x}}\left(t, x^{*}(t), \dot{x}^{*}(t)\right) T_{2}(t)=d$ for all $t \in[\underline{t}, \bar{t}]$.

In addition, there exist smooth functions

$$
\begin{array}{ll}
Z_{2}^{\prime}:[\underline{t}, \bar{t}] \rightarrow \mathbb{R}^{(\mu+1) n,(\mu+1) n-a}, & T_{1}:[\underline{t}, \bar{t}] \rightarrow \mathbb{R}^{(\mu+1) n, a} \\
T_{2}^{\prime}:[\underline{t}, \bar{t}] \rightarrow \mathbb{R}^{n, a}, & T_{1}^{\prime}:[\underline{t}, \bar{t}] \rightarrow \mathbb{R}^{(\mu+1) n,(\mu+1) n-a},
\end{array}
$$

such that the matrix valued functions $\left[Z_{2}^{\prime}, Z_{2}\right],\left[T_{1}^{\prime}, T_{1}\right]$ and $\left[T_{2}^{\prime}, T_{2}\right]$ are pointwise orthogonal and, furthermore,

$$
Z_{2}^{\prime}(t)^{T} F_{\mu ; \dot{x}, \ldots, x^{(\mu+1)}}\left(t, x^{*}(t), P(t)\right) T_{1}(t)=0 \quad \text { for all } t \in[\underline{t}, \bar{t}] .
$$

It has been shown in [10] that for every

$$
\left(t_{0}, x_{0}, \dot{x}_{0}, \ldots, x_{0}^{(\mu+1)}\right) \in \mathbb{L}_{\mu}
$$

the DAE (1.1a), if it satisfies Hypothesis 2.1, then it locally defines a function $x$ from a neighborhood of $t_{0}$ into $\mathbb{R}^{n}$. In particular, this $x$ solves a DAE of differentiation index at most one that is extracted from the derivative array equations $F_{\mu}\left(t, x, \dot{x}, \ldots, x^{(\mu+1)}\right)=0$. This solution can be extended until the boundary of the set where $F_{\mu}$ is defined is reached. Since

$$
\left(t_{0}, x^{*}\left(t_{0}\right), P\left(t_{0}\right)\right) \in \mathbb{L}_{\mu}, \quad t_{0} \in[\underline{t}, \bar{t}]
$$

defines a solution on $[\underline{t}, \bar{t}]$, the same holds for every $\left(t_{0}, x_{0}, y_{0}\right) \in \mathbb{L}_{\mu}$ in a neighborhood of $\left(t_{0}, x^{*}\left(t_{0}\right), P\left(t_{0}\right)\right)$.

In this section we have briefly presented some results on the solution and formulation of general nonlinear systems of differential-algebraic equations. In the next section we use these results to analyze the single shooting method and the local uniqueness of solutions to the resulting nonlinear systems.

\section{Single shooting and local uniqueness}

In this section, which is of a more theoretical nature, we discuss the single shooting method. If initial value problems are uniquely solvable, then we can see the value of the solution at a certain point as a function of the initial value. This means that also the boundary condition of a boundary value problem becomes a function of the initial value. Therefore, a solution of a boundary value problem is said to be locally unique if the corresponding initial value is a locally unique solution of the boundary condition. For DAEs we must of course take into account that an initial condition must be consistent in the sense that there is a related point in the set $\mathbb{L}_{\mu}$.

For this reason we locally define a (nonlinear) projection onto $\mathbb{L}_{\mu}$ by considering the nonlinear system

$$
\begin{array}{ll}
\text { (a) } & F_{\mu}(\underline{t}, \hat{x}, \hat{y})=0, \\
\text { (b) } & T_{2}(\underline{t})^{T}(\hat{x}-x)=0, \\
\text { (c) } & T_{1}(\underline{t})^{T}(\hat{y}-y)=0,
\end{array}
$$


in the unknowns $(x, y, \hat{x}, \hat{y})$.

If we write (3.1) as

$$
H(x, y, \hat{x}, \hat{y})=0
$$

then a solution of this system is given by $\left(x^{*}(\underline{t}), P(\underline{t}), x^{*}(\underline{t}), P(\underline{t})\right)$. Since the Jacobian with respect to $\hat{x}, \hat{y}$ satisfies

$$
\begin{aligned}
& \operatorname{rank} H_{\hat{x}, \hat{y}}\left(x^{*}(\underline{t}), P(\underline{t}), x^{*}(\underline{t}), P(\underline{t})\right)= \\
&=\operatorname{rank}\left[\begin{array}{cc}
F_{\mu ; x}\left(\underline{t}, x^{*}(\underline{t}), P(\underline{t})\right) & F_{\mu ; y}\left(\underline{t}, x^{*}(\underline{t}), P(\underline{t})\right) \\
T_{2}(\underline{t})^{T} & 0 \\
0 & T_{1}(\underline{t})^{T}
\end{array}\right]= \\
&=\operatorname{rank}\left[\begin{array}{cc}
Z_{2}^{\prime}(\underline{t})^{T} F_{\mu ; x}\left(\underline{t}, x^{*}(\underline{t}), P(\underline{t})\right) & Z_{2}^{\prime}(\underline{t})^{T} F_{\mu ; y}\left(\underline{t}, x^{*}(\underline{t}), P(\underline{t})\right) \\
Z_{2}(\underline{t})^{T} F_{\mu ; x}\left(\underline{t}, x^{*}(\underline{t}), P(\underline{t})\right) & 0 \\
T_{2}(\underline{t})^{T} & 0 \\
0 & T_{1}(\underline{t})^{T}
\end{array}\right],
\end{aligned}
$$

and since by construction the matrices

$$
\left[\begin{array}{c}
Z_{2}^{\prime}(t)^{T} F_{\mu ; y}\left(t, x^{*}(t), P(t)\right) \\
T_{1}(t)^{T}
\end{array}\right],\left[\begin{array}{c}
Z_{2}(t)^{T} F_{\mu ; x}\left(t, x^{*}(t), P(t)\right) \\
T_{2}(t)^{T}
\end{array}\right]
$$

are nonsingular for all $t \in[\underline{t}, \bar{t}]$, it follows that $H_{\hat{x}, \hat{y}}\left(x^{*}(\underline{t}), P(\underline{t}), x^{*}(\underline{t}), P(\underline{t})\right)$ is nonsingular. We can therefore solve locally for $(\hat{x}, \hat{y})$ obtaining a function $S$ according to

$$
(\hat{x}, \hat{y})=S(x, y)
$$

Since $F_{\mu}(\underline{t}, S(x, y))=0$, we have that $(\underline{t}, S(x, y)) \in \mathbb{L}_{\mu}$ for every $(x, y)$ in a neighborhood of $\left(x^{*}(\underline{t}), P(\underline{t})\right)$. Observing that the initial value problem for (1.1a) together with $\left(\underline{t}, S\left(x^{*}(\underline{t}), P(\underline{t})\right)\right) \in \mathbb{L}_{\mu}$ is solvable on the whole interval $[\underline{t}, \bar{t}]$, the initial value problem remains solvable on the whole interval $[\underline{t}, \bar{t}]$ with an initial condition given by $(\underline{t}, \underline{x}, \underline{y})$ from a neighborhood $\mathbb{L}_{\mu} \cap \mathbb{U}$ of $\left(\underline{t}, x^{*}(\underline{t}), P(\underline{t})\right)$. Thus the DAE defines a flow

$$
\Phi: \mathbb{V} \rightarrow \mathbb{R}^{n}, \quad \mathbb{V}=\left\{(\underline{x}, \underline{y}) \mid(\underline{t}, \underline{x}, \underline{y}) \in \mathbb{L}_{\mu} \cap \mathbb{U}\right\}
$$

that maps $(\underline{x}, \underline{y}) \in \mathbb{V}$ on the final value $x(\bar{t})$ of the solution $x$ of the associated initial value problem.

For later use, we will need the derivatives of $S$ at $\left(x^{*}(\underline{t}), P(\underline{t})\right)$. These are given by

$$
H_{\hat{x}, \hat{y}}\left(x^{*}(\underline{t}), P(\underline{t}), x^{*}(\underline{t}), P(\underline{t})\right) S_{x, y}\left(x^{*}(\underline{t}), P(\underline{t})\right)=-H_{x, y}\left(x^{*}(\underline{t}), P(\underline{t}), x^{*}(\underline{t}), P(\underline{t})\right),
$$

i. e.,

$$
\left[\begin{array}{cc}
F_{\mu, x}\left(\underline{t}, x^{*}(\underline{t}), P(\underline{t})\right) & F_{\mu ; y}\left(\underline{t}, x^{*}(\underline{t}), P(\underline{t})\right) \\
T_{2}(\underline{t})^{T} & 0 \\
0 & T_{1}(\underline{t})^{T}
\end{array}\right] S_{x, y}\left(x^{*}(\underline{t}), P(\underline{t})\right)=\left[\begin{array}{cc}
0 & 0 \\
T_{2}(\underline{t})^{T} & 0 \\
0 & T_{1}(\underline{t})^{T}
\end{array}\right] .
$$

Let the columns of $W$ be orthonormal and span kernel $F_{\mu ; x, y}\left(\underline{t}, x^{*}(\underline{t}), P(\underline{t})\right)$. Setting

$$
\tilde{W}=\left[\begin{array}{cc}
T_{2}(\underline{t}) & 0 \\
0 & T_{1}(\underline{t})
\end{array}\right]
$$


we see that the matrix $\tilde{W}^{T} W$ is nonsingular, since $H_{\hat{x}, \hat{y}}\left(x^{*}(\underline{t}), P(\underline{t}), x^{*}(\underline{t}), P(\underline{t})\right)$ is nonsingular, and we have

$$
S_{x, y}\left(x^{*}(\underline{t}), P(\underline{t})\right)=W\left(\tilde{W}^{T} W\right)^{-1} \tilde{W}^{T} .
$$

We then have the following theorem on the local uniqueness of solutions of boundary value problems for differential-algebraic equations.

Theorem 3.1 The function $x^{*}$ in (2.3) is a locally unique solution of the boundary value problem (1.1) in the sense that $\left(x^{*}(\underline{t}), P(\underline{t})\right)$ is a solution of

$$
\begin{array}{ll}
\text { (a) } & F_{\mu}(\underline{t}, \underline{x}, \underline{y})=0, \\
\text { (b) } & T_{1}(\underline{t})^{T}(\underline{y}-P(\underline{t}))=0, \\
\text { (c) } & r(\underline{x}, \Phi(S(\underline{x}, \underline{y})))=0,
\end{array}
$$

with nonsingular Jacobian if and only if

$$
\mathcal{E}=C T_{2}(\underline{t})+D \Phi_{x, y}\left(x^{*}(\underline{t}), P(\underline{t})\right) S_{x}\left(x^{*}(\underline{t}), P(\underline{t})\right) T_{2}(\underline{t})
$$

is nonsingular, where $C=r_{x_{a}}\left(x^{*}(\underline{t}), x^{*}(\bar{t})\right)$ and $D=r_{x_{b}}\left(x^{*}(\underline{t}), x^{*}(\bar{t})\right)$.

Proof. Obviously, $(\underline{x}, \underline{y})=\left(x^{*}(\underline{t}), P(\underline{t})\right)$ is a solution of (3.7). Moreover, the Jacobian $J$ of (3.7) is given by

$$
J=\left[\begin{array}{cc}
F_{\mu ; x}\left(\underline{t}, x^{*}(\underline{t}), P(\underline{t})\right) & F_{\mu ; y}\left(\underline{t}, x^{*}(\underline{t}), P(\underline{t})\right) \\
0 & T_{1}(\underline{t})^{T} \\
C+D \Phi_{x, y}\left(x^{*}(\underline{t}), P(\underline{t})\right) S_{x}\left(x^{*}(\underline{t}), P(\underline{t})\right) & D \Phi_{x, y}\left(x^{*}(\underline{t}), P(\underline{t})\right) S_{y}\left(x^{*}(\underline{t}), P(\underline{t})\right)
\end{array}\right] .
$$

Omitting arguments, we have that

$$
\operatorname{rank} J=\operatorname{rank}\left[\begin{array}{cccc}
Z_{2}^{\prime T} F_{\mu ; x} T_{2}^{\prime} & Z_{2}^{\prime T} F_{\mu ; x} T_{2} & Z_{2}^{\prime T} F_{\mu ; y} T_{1}^{\prime} & 0 \\
Z_{2}^{T} F_{\mu ; x} T_{2}^{\prime} & 0 & 0 & 0 \\
0 & 0 & 0 & I \\
\left(C+D \Phi_{x, y} S_{x}\right) T_{2}^{\prime} & \left(C+D \Phi_{x, y} S_{x}\right) T_{2} & D \Phi_{x, y} S_{y} T_{1}^{\prime} & D \Phi_{x, y} S_{y} T_{1}
\end{array}\right] .
$$

Since

$$
S_{x}=W\left(\tilde{W}^{T} W\right)^{-1}\left[\begin{array}{c}
T_{2}^{T} \\
0
\end{array}\right], \quad S_{y}=W\left(\tilde{W}^{T} W\right)^{-1}\left[\begin{array}{c}
0 \\
T_{1}^{T}
\end{array}\right]
$$

by (3.5), we have $S_{x} T_{2}^{\prime}=0$ and $S_{y} T_{1}^{\prime}=0$ by (2.6). Moreover, $Z_{2}^{T} F_{\mu ; x} T_{2}^{\prime}$ and $Z_{2}^{\prime T} F_{\mu ; y} T_{1}^{\prime}$ are nonsingular by construction. Thus $J$ has full rank if and only if

$$
\mathcal{E}=\left(C+D \Phi_{x, y} S_{x}\right) T_{2}
$$

has full rank.

Remark 3.2 In the case of linear boundary value problems, i. e., problems (1.1) where $F$ and $r$ are linear, the condition (3.8) coincides with that given in [14, 20, 21] and thus yields global uniqueness of the solution $x^{*}$

Proof. Since we do not need this result in the further course of this paper, we give the proof in the appendix. 


\section{Multiple shooting}

It is well known that in single shooting one is faced with the difficulty that the arising initial value problems may be unstable. This may lead to large solution components or even to the problem that the solution does not extend until $\bar{t}$ due to errors in the initial guess. To overcome these difficulties, in multiple shooting the solution interval is therefore split beforehand into smaller subintervals according to

$$
\underline{t}=t_{0}<t_{1}<\cdots<t_{N-1}<t_{N}=\bar{t}, \quad N \in \mathbb{N} .
$$

Given initial guesses

$$
\left(x_{i}, y_{i}\right) \in \mathbb{R}^{(\mu+2) n}, \quad i=0, \ldots, N,
$$

at these points, the idea is to project $\left(t_{i}, x_{i}, y_{i}\right)$ onto $\mathbb{L}_{\mu}$ and to solve the associated initial value problems on $\left[t_{i}, t_{i+1}\right]$, requiring that the pieces match to a continuous solution on the whole interval and that the boundary condition is satisfied.

In contrast to Section 3 which was merely dedicated to a theoretical investigation, in this section we present a method that can actually be implemented. We therefore are not allowed to use functions as $Z_{2}$ or $T_{2}$ in the definition of the procedure. Instead, we must look for computationally available quantities.

Given $\left(t_{i}, x_{i}, y_{i}\right)$ as initial guess for a point on the set $\mathbb{L}_{\mu}$, we can solve $F_{\mu}\left(t_{i}, x, y\right)=0$ by the Gauß-Newton method (see, e. g., [16]) to obtain $\left(t_{i}, \tilde{x}_{i}, \tilde{y}_{i}\right) \in \mathbb{L}_{\mu}$. Of course, we must require that the guess $\left(t_{i}, x_{i}, y_{i}\right)$ is good enough to guarantee convergence. Applying Hypothesis 2.1, we can then compute quantities $\tilde{Z}_{2, i}$ and $\tilde{T}_{2, i}$ where the columns form orthonormal bases of corange $F_{\mu ; y}\left(t_{i}, \tilde{x}_{i}, \tilde{y}_{i}\right)$ and kernel $\tilde{Z}_{2, i}^{T} F_{\mu ; x}\left(t_{i}, \tilde{x}_{i}, \tilde{y}_{i}\right)$, respectively. In the same way, we can determine quantities $\tilde{Z}_{2, i}^{\prime}$ and $\tilde{T}_{1, i}$.

Similar to (3.1), the system

$$
\begin{aligned}
& \text { (a) } \quad F_{\mu}\left(t_{i}, \hat{x}_{i}, \hat{y}_{i}\right)=0, \\
& \text { (b) } \tilde{T}_{2, i}^{T}\left(\hat{x}_{i}-x_{i}\right)=0, \\
& \text { (c) } \tilde{T}_{1, i}^{T}\left(\hat{y}_{i}-y_{i}\right)=0
\end{aligned}
$$

locally defines functions $S_{i}$ according to

$$
\left(\hat{x}_{i}, \hat{y}_{i}\right)=S_{i}\left(x_{i}, y_{i}\right)
$$

in such a way that $\left(t_{i}, S_{i}\left(x_{i}, y_{i}\right)\right) \in \mathbb{L}_{\mu}$. Defining $W_{i}$ to have columns that form an orthonormal basis of kernel $F_{\mu ; x, y}\left(t_{i}, \hat{x}_{i}, \hat{y}_{i}\right)$ with $\left(t_{i}, \hat{x}_{i}, \hat{y}_{i}\right) \in \mathbb{L}_{\mu}$ and setting

$$
\tilde{W}_{i}=\left[\begin{array}{cc}
\tilde{T}_{2, i} & 0 \\
0 & \tilde{T}_{1, i}
\end{array}\right]
$$

we obtain

$$
S_{i ; x, y}\left(\hat{x}_{i}, \hat{y}_{i}\right)=W_{i}\left(\tilde{W}_{i}^{T} W_{i}\right)^{-1} \tilde{W}_{i}^{T}
$$

similar to (3.6) as long as $\tilde{W}_{i}^{T} W_{i}$ is invertible. In the same way as done with $\Phi$ in Section 3 , we define flows $\Phi_{i}$ that map initial values $\left(\hat{x}_{i}, \hat{y}_{i}\right)$ with $\left(t_{i}, \hat{x}_{i}, \hat{y}_{i}\right) \in \mathbb{L}_{\mu}$ on the value $x\left(t_{i+1}\right)$ of the solution $x$ of the corresponding initial value problem. 
The multiple shooting system then is given by

$$
\begin{array}{lll}
\text { (a) } & F_{\mu}\left(t_{i}, x_{i}, y_{i}\right)=0, & i=0, \ldots, N \\
\text { (b) } & \tilde{T}_{2, i+1}^{T}\left(x_{i+1}-\Phi_{i}\left(S_{i}\left(x_{i}, y_{i}\right)\right)\right)=0, & i=0, \ldots, N-1, \\
\text { (c) } & r\left(x_{0}, x_{N}\right)=0 . &
\end{array}
$$

Comparing with the single shooting method of Section 3, (3.7a) is now required in (4.7a) at all mesh points $t_{i}$ with corresponding unknowns $\left(x_{i}, y_{i}\right)$. Besides the boundary condition (4.7c), we impose continuity conditions for the differential components in (4.7b). Condition (3.7b), which was responsible for local uniqueness of the solution in (3.7), cannot be used here because it involves knowledge of the actual solution. Thus, in the present form, system (4.7) is underdetermined. It is therefore solved by a Gauß-Newton-type iteration method which we present in the following. In the course of the presentation, we will select a suitable generalized inverse of the Jacobian by additional conditions which will turn out to be the appropriate replacement for $(3.7 \mathrm{~b})$.

Given approximations $\left(x_{i}, y_{i}\right)$, the Gauß-Newton-type method is defined by the corrections $\left(\Delta x_{i}, \Delta y_{i}\right)$ that are added to $\left(x_{i}, y_{i}\right)$ to get updated approximations. In the (underdetermined) ordinary Gauß-Newton method, these corrections satisfy the linearized equations

$$
\begin{gathered}
\text { (a) } F_{\mu ; x}\left(t_{i}, x_{i}, y_{i}\right) \Delta x_{i}+F_{\mu ; y}\left(t_{i}, x_{i}, y_{i}\right) \Delta y_{i}=-F_{\mu}\left(t_{i}, x_{i}, y_{i}\right) \\
\text { (b) } \tilde{T}_{2, i+1}^{T}\left(\Delta x_{i+1}-\Phi_{i ; x, y}\left(S_{i}\left(x_{i}, y_{i}\right)\right)\left(S_{i ; x}\left(x_{i}, y_{i}\right) \Delta x_{i}+S_{i ; y}\left(x_{i}, y_{i}\right) \Delta y_{i}\right)\right)= \\
=-\tilde{T}_{2, i+1}^{T}\left(x_{i+1}-\Phi_{i}\left(S_{i}\left(x_{i}, y_{i}\right)\right)\right), \\
\text { (c) } r_{x_{a}}\left(x_{0}, x_{N}\right) \Delta x_{0}+r_{x_{b}}\left(x_{0}, x_{N}\right) \Delta x_{N}=-r\left(x_{0}, x_{N}\right) .
\end{gathered}
$$

For an efficient numerical method, however, the structure and the properties of the Jacobian should be utilized. In the following, we will perturb the coefficient matrix in such a way that the system decouples into smaller systems of reasonable size. In particular, the perturbations that we apply will tend to zero when the $\left(x_{i}, y_{i}\right)$ converge to a solution of (4.7) resulting in a Gauß-Newton-like process with superlinear convergence rate, cp. [4].

In a solution of (4.7), the matrices $F_{\mu ; y}\left(t_{i}, x_{i}, y_{i}\right)$ will have rank deficiency $a$. We therefore perturb $F_{\mu ; y}\left(t_{i}, x_{i}, y_{i}\right)$ to matrices $\tilde{M}_{i}$ with rank deficiency $a$. The only condition we must require is that these perturbations tend to zero when the matrices $F_{\mu ; y}\left(t_{i}, x_{i}, y_{i}\right)$ tend to matrices with rank deficiency $a$. One possibility to achieve this is to neglect the $a$ smallest singular values of $F_{\mu ; y}\left(t_{i}, x_{i}, y_{i}\right)$, see, e. g., [6]. The equations (4.8a) are thus replaced by

$$
F_{\mu ; x}\left(t_{i}, x_{i}, y_{i}\right) \Delta x_{i}+\tilde{M}_{i} \Delta y_{i}=-F_{\mu}\left(t_{i}, x_{i}, y_{i}\right) .
$$

Let the columns of $Z_{2, i}$ form an orthonormal basis of corange $\tilde{M}_{i}$ and let $\left[Z_{2, i}^{\prime}, Z_{2, i}\right]$ be orthogonal. Relation (4.9) then splits into

$$
\begin{array}{ll}
\text { (a) } & Z_{2, i}^{\prime T} F_{\mu ; x}\left(t_{i}, x_{i}, y_{i}\right) \Delta x_{i}+Z_{2, i}^{\prime T} \tilde{M}_{i} \Delta y_{i}=-Z_{2, i}^{\prime T} F_{\mu}\left(t_{i}, x_{i}, y_{i}\right), \\
\text { (b) } & Z_{2, i}^{T} F_{\mu ; x}\left(t_{i}, x_{i}, y_{i}\right) \Delta x_{i}=-Z_{2, i}^{T} F_{\mu}\left(t_{i}, x_{i}, y_{i}\right) .
\end{array}
$$

Requiring in addition that

$$
\tilde{T}_{1, i}^{T} \Delta y_{i}=0
$$

as substitute for $(3.7 \mathrm{~b})$ and observing that

$$
\left[\begin{array}{c}
Z_{2, i}^{\prime T} \tilde{M}_{i} \\
\tilde{T}_{1, i}^{T}
\end{array}\right]
$$


is nonsingular for sufficiently good initial guesses $\left(x_{i}, y_{i}\right)$, it follows that we can solve (4.10a) with (4.11) for $\Delta y_{i}$ in terms of $\Delta x_{i}$.

Let the columns of $T_{2, i}$ form an orthonormal basis of kernel $Z_{2, i}^{T} F_{\mu ; x}\left(t_{i}, x_{i}, y_{i}\right)$. For sufficiently good initial guesses $\left(x_{i}, y_{i}\right)$ also $\tilde{T}_{2, i}^{T} T_{2, i}$ is nonsingular. Thus, there exists a matrix $T_{2, i}^{\prime}$ such that $\left[T_{2, i}^{\prime}, T_{2, i}\right]$ is nonsingular and

$$
\tilde{T}_{2, i}^{T} T_{2, i}^{\prime}=0
$$

Defining $\Delta v_{i}^{\prime}$ and $\Delta v_{i}$ by the relation

$$
\Delta x_{i}=T_{2, i}^{\prime} \Delta v_{i}^{\prime}+T_{2, i} \Delta v_{i}
$$

equation (4.10b) becomes

$$
Z_{2, i}^{T} F_{\mu ; x}\left(t_{i}, x_{i}, y_{i}\right) T_{2, i}^{\prime} \Delta v_{i}^{\prime}=-Z_{2, i}^{T} F_{\mu}\left(t_{i}, x_{i}, y_{i}\right) .
$$

Since $Z_{2, i}^{T} F_{\mu ; x}\left(t_{i}, x_{i}, y_{i}\right) T_{2, i}^{\prime}$ is nonsingular by construction, (4.14) can be solved for $\Delta v_{i}^{\prime}$.

Turning to $(4.8 \mathrm{~b})$ we know that at a solution of (4.8) the relations

$$
\begin{aligned}
& \text { (a) } S_{i ; x}\left(x_{i}, y_{i}\right) \Delta x_{i}=W_{i}\left(\tilde{W}_{i}^{T} W_{i}\right)^{-1}\left[\begin{array}{c}
\tilde{T}_{2, i}^{T} \\
0
\end{array}\right] T_{2, i} \Delta v_{i}=S_{i ; x}\left(x_{i}, y_{i}\right) T_{2, i} \Delta v_{i}, \\
& \text { (b) } S_{i ; y}\left(x_{i}, y_{i}\right) \Delta y_{i}=W_{i}\left(\tilde{W}_{i}^{T} W_{i}\right)^{-1}\left[\begin{array}{c}
0 \\
\tilde{T}_{1, i}^{T}
\end{array}\right] \Delta y_{i}=0
\end{aligned}
$$

hold because of (4.11) and (4.12). Thus, we replace (4.8b) by

$$
\begin{gathered}
\tilde{T}_{2, i+1}^{T} T_{2, i+1} \Delta v_{i+1}-\tilde{T}_{2, i+1}^{T} \Phi_{i ; x, y}\left(S_{i}\left(x_{i}, y_{i}\right)\right) S_{i ; x}\left(x_{i}, y_{i}\right) T_{2, i} \Delta v_{i}= \\
=-\tilde{T}_{2, i+1}^{T}\left(x_{i+1}-\Phi_{i}\left(S_{i}\left(x_{i}, y_{i}\right)\right)\right),
\end{gathered}
$$

which is again a perturbation that tends to zero when the iteration converges. The main advantage of (4.16) is that we only need the derivative $\Phi_{i ; x, y}\left(S_{i}\left(x_{i}, y_{i}\right)\right) S_{i ; x}\left(x_{i}, y_{i}\right)$ in the direction of the $d$ columns of $T_{2, i}$. In particular, if we use numerical differentiation to approximate this derivative, then we only need to solve $d$ initial value problems.

Finally, we write $(4.8 \mathrm{c})$ in the form

$$
\begin{aligned}
& r_{x_{a}}\left(x_{0}, x_{N}\right) T_{2,0} \Delta v_{0}+r_{x_{b}}\left(x_{0}, x_{N}\right) T_{2, N} \Delta v_{N}= \\
& \quad=-r\left(x_{0}, x_{N}\right)-r_{x_{a}}\left(x_{0}, x_{N}\right) T_{2,0}^{\prime} \Delta v_{0}^{\prime}-r_{x_{b}}\left(x_{0}, x_{N}\right) T_{2, N}^{\prime} \Delta v_{N}^{\prime} .
\end{aligned}
$$

Setting

$$
\begin{aligned}
& \text { (a) } G_{i}=\tilde{T}_{2, i+1}^{T} \Phi_{i ; x, y}\left(S_{i}\left(x_{i}, y_{i}\right)\right) S_{i ; x}\left(x_{i}, y_{i}\right) T_{2, i}, \quad i=0, \ldots, N-1, \\
& \text { (b) } J_{i}=\tilde{T}_{2, i}^{T} T_{2, i}, \quad i=1, \ldots, N \\
& \text { (c) } \tilde{C}=r_{x_{a}}\left(x_{0}, x_{N}\right) T_{2,0}, \quad \tilde{D}=r_{x_{b}}\left(x_{0}, x_{N}\right) T_{2, N}
\end{aligned}
$$

the linear system that we have to solve for the unknowns $\Delta v_{i}$ has the shooting-like coefficient matrix

$$
\tilde{\mathcal{E}}_{N}=\left[\begin{array}{ccccc}
-G_{0} & J_{1} & & & \\
& -G_{1} & J_{2} & & \\
& & \ddots & \ddots & \\
\tilde{C} & & & -G_{N-1} & J_{N} \\
\tilde{D}
\end{array}\right] .
$$


This system can be solved by standard methods such as Gaussian elimination with pivoting [6]. Since the blocks $J_{i}$ are invertible for sufficiently good initial guesses $\left(x_{i}, y_{i}\right)$, it follows that the matrix $\tilde{\mathcal{E}}_{N}$ is nonsingular if and only if

$$
\mathcal{E}_{N}=\tilde{C}+\tilde{D}\left(J_{N}^{-1} G_{N-1}\right)\left(J_{N-1}^{-1} G_{N-2}\right) \cdots\left(J_{2}^{-1} G_{1}\right)\left(J_{1}^{-1} G_{0}\right)
$$

is nonsingular. Thus, for the method to work, it suffices to show that this is the case at least at the solution and therefore in some neighborhood of it.

At a solution $\left(x_{i}, y_{i}\right)=\left(x^{*}\left(t_{i}\right), y_{i}^{*}\right)$, the matrix $\mathcal{E}_{N}$ takes the form

$$
\mathcal{E}_{N}=C T_{2,0}+D T_{2, N} \prod_{i=N-1}^{i=0}\left[\left(\tilde{T}_{2, i+1}^{T} T_{2, i+1}\right)^{-1} \tilde{T}_{2, i+1}^{T} \Phi_{i ; x, y}\left(S_{i}\left(x_{i}, y_{i}\right)\right) S_{i ; x}\left(x_{i}, y_{i}\right) T_{2, i}\right] .
$$

To take into account that $\Phi_{i}\left(S_{i}(x, y)\right)$ is consistent at $t_{i+1}$ for $(x, y)$ in a neighborhood of $\left(x_{i}, y_{i}\right)$ as the value of a solution of the DAE on $\left[t_{i}, t_{i+1}\right]$, we consider the system

$$
\begin{array}{ll}
\text { (a) } & F_{\mu}\left(t_{i}, x, \hat{y}\right)+Z_{2, i} \alpha=0, \\
\text { (b) } & \tilde{T}_{1, i}^{T}\left(\hat{y}-y_{i}\right)=0 .
\end{array}
$$

Writing this as

$$
H_{i}(x, \hat{y}, \alpha)=0
$$

we know that $H_{i}\left(x_{i}, y_{i}, 0\right)=0$. Since

$$
\begin{aligned}
& \operatorname{rank} H_{i ; \hat{y}, \alpha}\left(x_{i}, y_{i}, 0\right)= \\
& \quad=\operatorname{rank}\left[\begin{array}{cc}
F_{\mu ; y}\left(t_{i}, x_{i}, y_{i}\right) & Z_{2, i} \\
\tilde{T}_{1, i}^{T} & 0
\end{array}\right]=\operatorname{rank}\left[\begin{array}{cc}
Z_{2, i}^{\prime T} F_{\mu ; y}\left(t_{i}, x_{i}, y_{i}\right) & 0 \\
0 & I \\
\tilde{T}_{1, i}^{T} & 0
\end{array}\right],
\end{aligned}
$$

the construction of $Z_{2, i}^{\prime}$ and $\tilde{T}_{1, i}$ guarantees that the matrix $H_{i ; \hat{y}, \alpha}\left(x_{i}, y_{i}, 0\right)$ is nonsingular. Thus, (4.22) locally defines functions $K_{i}$ and $L_{i}$ according to

$$
\hat{y}=K_{i}(x), \quad \alpha=L_{i}(x) .
$$

For all $x$ with $L_{i}(x)=0$ we have $F_{\mu}\left(t_{i}, x, K_{i}(x)\right)=0$ and $x$ is consistent at $t_{i}$. Furthermore, differentiating

$$
F_{\mu}\left(t_{i}, x, K_{i}(x)\right)+Z_{2, i} L_{i}(x)=0,
$$

evaluating at $x_{i}$ and multiplying by $Z_{2, i}^{T}$ yields

$$
L_{i ; x}\left(x_{i}\right)=-Z_{2, i}^{T} F_{\mu ; x}\left(t_{i}, x_{i}, y_{i}\right) .
$$

Hence, $L_{i ; x}$ has full row rank in a neighborhood of $x_{i}$ and all solutions of $L_{i}(x)=0$ form a manifold of dimension $d=n-a$ which is a submanifold of the manifold of consistent values at point $t_{i}$. Since the dimension of the latter manifold is also $d$, see [10], they must coincide.

Thus, given an $x$ that is consistent at $t_{i}$, the function $K_{i}$ yields a $\hat{y}$ such that $\left(t_{i}, x, \hat{y}\right) \in \mathbb{L}_{\mu}$ while $L_{i}(x)=0$. In particular,

$$
F_{\mu}\left(t_{i+1}, \Phi_{i}\left(S_{i}(x, y)\right), K_{i+1}\left(\Phi_{i}\left(S_{i}(x, y)\right)\right)\right)=0
$$


holds in a neighborhood of $\left(x_{i}, y_{i}\right)$. Differentiating this relation with respect to $(x, y)$ and setting $(x, y)=\left(x_{i}, y_{i}\right)$, we obtain

$$
\begin{aligned}
& F_{\mu ; x}\left(t_{i+1}, x_{i+1}, y_{i+1}\right) \Phi_{i ; x, y}\left(S_{i}\left(x_{i}, y_{i}\right)\right) S_{i ; x, y}\left(x_{i}, y_{i}\right)+ \\
& \quad+F_{\mu ; y}\left(t_{i+1}, x_{i+1}, y_{i+1}\right) K_{i+1 ; x}\left(x_{i+1}\right) \Phi_{i ; x, y}\left(S_{i}\left(x_{i}, y_{i}\right)\right) S_{i ; x, y}\left(x_{i}, y_{i}\right)=0
\end{aligned}
$$

Multiplying with $Z_{2, i+1}^{T}$ from the left finally yields

$$
Z_{2, i+1}^{T} F_{\mu ; x}\left(t_{i+1}, x_{i+1}, y_{i+1}\right) \Phi_{i ; x, y}\left(S_{i}\left(x_{i}, y_{i}\right)\right) S_{i ; x, y}\left(x_{i}, y_{i}\right)=0
$$

Hence, the columns of $\Phi_{i ; x, y}\left(S_{i}\left(x_{i}, y_{i}\right)\right) S_{i ; x, y}\left(x_{i}, y_{i}\right)$ lie in the kernel of the matrix $Z_{2, i+1}^{T} F_{\mu ; x}\left(t_{i+1}, x_{i+1}, y_{i+1}\right)$ which in turn is spanned by the columns of $T_{2, i+1}$. Since the expression $T_{2, i+1}\left(\tilde{T}_{2, i+1}^{T} T_{2, i+1}\right)^{-1} \tilde{T}_{2, i+1}^{T}$ is a projector on this kernel, we have

$$
\begin{array}{r}
T_{2, i+1}\left(\tilde{T}_{2, i+1}^{T} T_{2, i+1}\right)^{-1} \tilde{T}_{2, i+1}^{T} \Phi_{i ; x, y}\left(S_{i}\left(x_{i}, y_{i}\right)\right) S_{i ; x, y}\left(x_{i}, y_{i}\right)= \\
=\Phi_{i ; x, y}\left(S_{i}\left(x_{i}, y_{i}\right)\right) S_{i ; x, y}\left(x_{i}, y_{i}\right) .
\end{array}
$$

Thus, (4.21) reduces to

$$
\mathcal{E}_{N}=C T_{2,0}+D\left[\prod_{i=N-1}^{i=0} \Phi_{i ; x, y}\left(S_{i}\left(x_{i}, y_{i}\right)\right) S_{i ; x}\left(x_{i}, y_{i}\right)\right] T_{2,0} .
$$

Finally, defining

$$
\Psi_{i}(x)=\left(x, K_{i}(x)\right)
$$

and using $\tilde{T}_{1, i}^{T} K_{i ; x}\left(x_{i}\right)=0$, which holds due to $(4.22 \mathrm{~b})$, we find that

$$
\begin{aligned}
& S_{i ; x, y}\left(x_{i}, y_{i}\right) \Psi_{i ; x}\left(x_{i}\right)= \\
& =W_{i}\left(\tilde{W}_{i}^{T} W_{i}\right)^{-1}\left[\begin{array}{cc}
\tilde{T}_{2, i}^{T} & 0 \\
0 & \tilde{T}_{1, i}^{T}
\end{array}\right]\left[\begin{array}{c}
I \\
K_{i ; x}\left(x_{i}\right)
\end{array}\right]= \\
& =W_{i}\left(\tilde{W}_{i}^{T} W_{i}\right)^{-1}\left[\begin{array}{c}
\tilde{T}_{2, i}^{T} \\
0
\end{array}\right]=S_{i ; x}\left(x_{i}, y_{i}\right) .
\end{aligned}
$$

Hence, (4.28) becomes

$$
\mathcal{E}_{N}=C T_{2,0}+D\left[\prod_{i=N-1}^{i=0} \Phi_{i ; x, y}\left(S_{i}\left(x_{i}, y_{i}\right)\right) S_{i ; x, y}\left(x_{i}, y_{i}\right) \Psi_{i ; x}\left(x_{i}\right)\right] T_{2,0} .
$$

Comparing with (3.8), the term in brackets in (4.30) is nothing else than the derivative $\Phi_{x, y} \tilde{S}_{x}$ of $\Phi \circ \tilde{S}$ decomposed according to

$$
\Phi \circ \tilde{S}=\left(\Phi_{N-1} \circ S_{N-1}\right) \circ\left(\Psi_{N-1} \circ \Phi_{N-2} \circ S_{N-2}\right) \circ \cdots \circ\left(\Psi_{1} \circ \Phi_{0} \circ S_{0}\right),
$$

where $\tilde{S}$ differs from $S$ by replacing $T_{1}(\underline{t}), T_{2}(\underline{t})$ with $\tilde{T}_{1,0}, \tilde{T}_{2,0}$ in (3.1). This means that for sufficiently good initial guesses, the matrix $\mathcal{E}_{N}$ is nonsingular when $\mathcal{E}$ of (3.8) is nonsingular, i. e., when there is a locally unique solution of the boundary value problem in the sense of Theorem 3.1.

Summarizing the obtained results, we have the following convergence theorem. 
Theorem 4.1 Suppose that the boundary value problem (1.1) satisfies Hypothesis 2.1 and that (1.1) has a locally unique solution according to Theorem 3.1. Then, for sufficiently good initial guesses, the iterates of the Gauß-Newton-like procedure developed in the course of this section converge superlinearly to a solution of (4.7).

Proof. Writing the Gauß-Newton-like procedure for (4.7) in the form

$$
z_{\nu+1}=z_{\nu}-\mathcal{A}_{\nu}^{-} \mathcal{F}\left(z_{\nu}\right)
$$

where $\mathcal{A}_{\nu}$ is the chosen perturbation of $\mathcal{F}_{z}\left(z_{\nu}\right)$ and $\mathcal{A}_{\nu}^{-}$denotes the chosen pseudoinverse due to $(4.11)$, we have

$$
\mathcal{A}_{\nu} \mathcal{A}_{\nu}^{-}=I
$$

since $\mathcal{A}_{\nu}^{-}$yields a solution of the perturbed linear system. Thus, we get

$$
\begin{aligned}
& z_{\nu+1}-z_{\nu}=-\mathcal{A}_{\nu}^{-} \mathcal{F}\left(z_{\nu}\right)= \\
&=-\mathcal{A}_{\nu}^{-}\left[\mathcal{F}\left(z_{\nu}\right)-\mathcal{F}\left(z_{\nu-1}\right)-\mathcal{A}_{\nu-1}\left(z_{\nu}-z_{\nu-1}\right)\right]= \\
&=-\mathcal{A}_{\nu}^{-}\left[\left.\mathcal{F}\left(z_{\nu-1}+s\left(z_{\nu}-z_{\nu-1}\right)\right)\right|_{s=0} ^{s=1}-\mathcal{F}_{z}\left(z_{\nu-1}\right)\left(z_{\nu}-z_{\nu-1}\right)-\right. \\
&\left.\quad \quad-\left(\mathcal{A}_{\nu-1}-\mathcal{F}_{z}\left(z_{\nu-1}\right)\right)\left(z_{\nu}-z_{\nu-1}\right)\right]= \\
&=-\mathcal{A}_{\nu}^{-}\left[\int_{0}^{1}\left(\mathcal{F}_{z}\left(z_{\nu-1}+s\left(z_{\nu}-z_{\nu-1}\right)\right)-\mathcal{F}_{z}\left(z_{\nu-1}\right)\right)\left(z_{\nu}-z_{\nu-1}\right) d s-\right. \\
&\left.\quad-\left(\mathcal{A}_{\nu-1}-\mathcal{F}_{z}\left(z_{\nu-1}\right)\right)\left(z_{\nu}-z_{\nu-1}\right)\right]
\end{aligned}
$$

Introducing constants $\beta, \gamma$ and $\delta_{\nu}$ according to

$$
\left\|\mathcal{A}_{\nu}^{-}\right\| \leq \beta, \quad\left\|\mathcal{F}_{z}(u)-\mathcal{F}_{z}(v)\right\| \leq \gamma\|u-v\|, \quad\left\|\mathcal{A}_{\nu}-\mathcal{F}_{z}\left(z_{\nu}\right)\right\|=\delta_{\nu}
$$

for some vector norm and its corresponding matrix norm (recalling that we assume sufficient smoothness for the data), we obtain the estimate

$$
\left\|z_{\nu+1}-z_{\nu}\right\| \leq \frac{1}{2} \beta \gamma\left\|z_{\nu}-z_{\nu-1}\right\|^{2}+\beta \delta_{\nu-1}\left\|z_{\nu}-z_{\nu-1}\right\|
$$

Since $\delta_{\nu} \rightarrow 0$ when $z_{\nu}$ converges to a solution, superlinear convergence follows as in [4].

Remark 4.2 As already mentioned, the main problem in the construction of shooting methods for DAEs is how to deal with inconsistent intermediate iterates. In the method presented here, we used (locally defined) nonlinear projections on $\mathbb{L}_{\mu}$ to get consistent initial values. A second possibility would have been to shift the manifold $\mathbb{L}_{\mu}$ in such a way that the given inconsistent iterate then lies in the shifted manifold. In the case of single shooting, if

$$
(\hat{x}, \hat{y})=S\left(x_{0}, y_{0}\right)
$$

then we would define

$$
\hat{\mathbb{L}}_{\mu}=\left\{(t, x, y) \in \mathbb{R}^{(\mu+2) n+1} \mid F_{\mu}\left(t, x-x_{0}+\hat{x}, y-y_{0}+\hat{y}\right)=0\right\}
$$

and solve the arising initial value problem with respect to $\hat{\mathbb{L}}_{\mu}$. The same idea is used by [18] in the form of so-called relaxed algebraic constraints when these are explicitly available. One can show that using the technique of shifting the manifold would yield a method with the same properties as the new method that we have presented. However, the method of shifting the manifold has the disadvantage that it requires to modify $F_{\mu}$ for the use in the initial value solver. 


\section{$5 \quad$ Numerical experiments}

The procedure of Section 4 has been implemented in FORTRAN in form of a research code. All rank decisions, computations of kernel and corange matrices and their complements as well as the solution of linear equations are performed on the basis of singular value decompositions. In particular, no concern was laid on efficiency questions. All computations were done on a SUN UltraSPARC workstation with $167 \mathrm{MHz}$ in IEEE double precision.

In all subsequent examples, the Gauß-Newton-like procedure of Theorem 4.1 was terminated as soon as $\left\|\Delta z_{\nu}\right\|_{2} \leq 10^{-5}$, where $\Delta z_{\nu}=z_{\nu+1}-z_{\nu}$. The entries $G_{i}$ of (4.19) were approximated by numerical differentiation according to

$$
\begin{aligned}
& G_{i} e_{j}=\frac{1}{\eta} \tilde{T}_{2, i+1}^{T}\left[\Phi_{i}\left(S_{i}\left(x_{i}+\eta T_{2, i} e_{j}, y_{i}\right)\right)-\Phi_{i}\left(x_{i}, y_{i}\right)\right] \\
& j=1, \ldots, d, i=0, \ldots, N-1,
\end{aligned}
$$

where $e_{j}$ is the $j$-th canonical basis vector of $\mathbb{R}^{d}$. We used the choice $\eta=10^{-7}$. All occuring initial value problems were solved with GENDA of [13] using the tolerance $10^{-5}$.

Example 5.1 In [7], the model of a periodically driven electronic amplifier is given. The equations with $n=5$ for the unknowns $\left(U_{1}, \ldots, U_{5}\right)$ read

$$
\begin{aligned}
& \left(U_{E}(t)-U_{1}\right) / R_{0}+C_{1}\left(\dot{U}_{2}-\dot{U}_{1}\right)=0, \\
& \left(U_{B}-U_{2}\right) / R_{2}-U_{2} / R_{1}+C_{1}\left(\dot{U}_{1}-\dot{U}_{2}\right)-0.01 f\left(U_{2}-U_{3}\right)=0, \\
& f\left(U_{2}-U_{3}\right)-U_{3} / R_{3}-C_{2} \dot{U}_{3}=0 \\
& \left(U_{B}-U_{4}\right) / R_{4}+C_{3}\left(\dot{U}_{5}-\dot{U}_{4}\right)-0.99 f\left(U_{2}-U_{3}\right)=0 \\
& -U_{5} / R_{5}+C_{3}\left(\dot{U}_{4}-\dot{U}_{5}\right)=0
\end{aligned}
$$

with

$$
\begin{aligned}
& U_{E}(t)=0.4 \sin (200 \pi t), \quad U_{B}=6, \\
& f(U)=10^{-6}(\exp (U / 0.026)-1), \\
& R_{0}=1000, \quad R_{1}=\cdots=R_{5}=9000, \\
& C_{1}=10^{-6}, \quad C_{2}=2 \cdot 10^{-6}, \quad C_{3}=3 \cdot 10^{-6} .
\end{aligned}
$$

The problem is known to satisfy Hypothesis 2.1 with $\mu=0, d=3$, and $a=2$. If we ask for the periodic response of the amplifier, we are led to the boundary conditions

$$
U_{l}(0)=U_{l}(0.01), \quad l=2,3,5,
$$

thus $\underline{t}=0$ and $\bar{t}=0.01$. We used $N=1$ and determined the initial guess for the unknowns $\left(x_{i}, y_{i}\right), i=0, \ldots, N$, by integration starting with

$$
\left(0, V_{1}, V_{1}, U_{B}, 0,0,0, V_{2}, 0,0\right) \in \mathbb{L}_{\mu},
$$

where $V_{1}=U_{B} \frac{R_{1}}{R_{1}+R_{2}}$ and $V_{2}=-\frac{V_{1}}{R_{3} C_{2}}$.

Then the presented method successfully computed a periodic solution. The behaviour of the Gauß-Newton-like method is given in Table 1. One component of the periodic response of the amplifier is shown in Figure 1. 
Table 1: Results for Example 5.1

\begin{tabular}{c|c}
$\nu$ & $\left\|\Delta z_{\nu}\right\|_{2}$ \\
\hline 0 & $0.339 \mathrm{D}+02$ \\
1 & $0.113 \mathrm{D}-02$ \\
2 & $0.107 \mathrm{D}-04$ \\
3 & $0.168 \mathrm{D}-08$
\end{tabular}

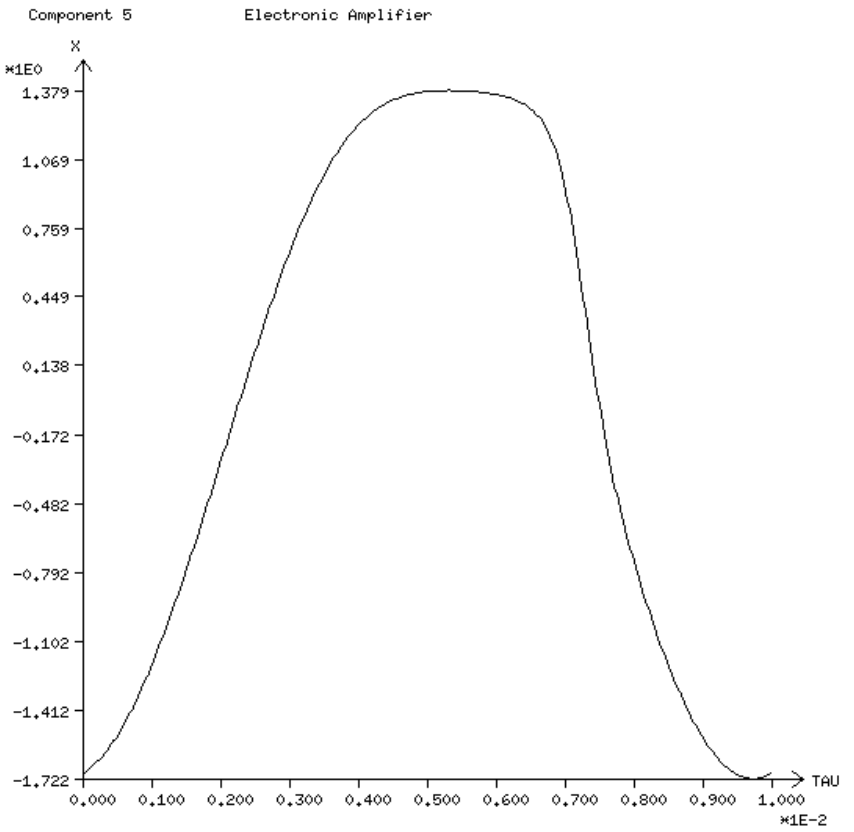

Figure 1: Periodic response for Example 5.1 
Table 2: Results for Example 5.2

\begin{tabular}{c|c}
$\nu$ & $\left\|\Delta z_{\nu}\right\|_{2}$ \\
\hline 0 & $0.727 \mathrm{D}+00$ \\
1 & $0.405 \mathrm{D}-01$ \\
2 & $0.345 \mathrm{D}-04$ \\
3 & $0.107 \mathrm{D}-09$
\end{tabular}

Example 5.2 In [17], a multibody system with non-holonomic constraint is presented. The model equations for the unknowns $\left(\varphi, z_{G}, z_{Z}, \lambda\right)$ are given by

$$
\begin{aligned}
& I_{R} \ddot{\varphi}=u, \\
& m_{G} \ddot{z}_{G}+d_{1}\left(\dot{z}_{G}-\dot{z}_{Z}\right)+c_{1}\left(z_{G}-z_{Z}\right)=\lambda, \\
& m_{Z} \ddot{z}_{Z}+d_{1}\left(\dot{z}_{Z}-\dot{z}_{G}\right)+c_{1}\left(z_{Z}-z_{G}\right)=0, \\
& \dot{z}_{G}=v_{U} \varphi
\end{aligned}
$$

where

$$
\begin{array}{lll}
I_{R}=0.002, & m_{G}=3, & m_{Z}=10 \\
v_{U}=2.8, & c_{1}=250, & d_{1}=10 .
\end{array}
$$

We ask for the time $T$ when starting from the trivial equilibrium the maximal allowed $\varphi_{\max }=$ 0.27 is reached for an external force $u=0.001$. The boundary conditions are then given by

$$
\varphi(0)=z_{G}(0)=z_{Z}(0)=\dot{\varphi}(0)=\dot{z}_{Z}(0)=0, \quad \varphi(T)=0.27 .
$$

Writing the model equations as first order system and transforming the unknown intervall $[0, T]$ to $[0,1]$, thus introducing $T$ as further unknown, we get a problem with $n=8$ in the unknowns $\left(\varphi, z_{G}, z_{Z}, \dot{\varphi}, \dot{z}_{G}, \dot{z}_{Z}, \lambda, T\right)$. Hypothesis 2.1 is here satisfied with $\mu=1, d=6$, and $a=2$. We set $N=1$ and took $T=1$ to get initial guesses for $\left(x_{i}, y_{i}\right)$ by integration starting with the equilibrium state. Table 2 shows the behaviour of the Gauß-Newton-like procedure.

Example 5.3 A pendulum in two space dimensions is modelled by

$$
\begin{aligned}
& \dot{p}_{1}=v_{1}, \quad \dot{v}_{1}=2 p_{1} \lambda, \\
& \dot{p}_{2}=v_{2}, \quad \dot{v}_{2}=2 p_{2} \lambda-g, \\
& p_{1}^{2}+p_{2}^{2}=1
\end{aligned}
$$

with the gravity constant $g=9.81$. The unknowns are $\left(p_{1}, p_{2}, v_{1}, v_{2}, \lambda\right)$. In [15] this problem together with the boundary conditions

$$
v_{2}(0)=0, \quad p_{1}(0.55)=0
$$

was used to test an implementation of a multiple shooting method for DAEs with $\mu=1$. Since for the above formulation we have $\mu=2$ together with $d=2$ and $a=3$, in [15] it was necessary to replace the constraint by its differentiated form

$$
2 p_{1} \dot{p}_{1}+2 p_{2} \dot{p}_{2}=2 p_{1} v_{1}+2 p_{2} v_{2}=0
$$


Table 3: Results for Example 5.3

Boundary condition
\begin{tabular}{c|c}
$\nu$ & $\left\|\Delta z_{\nu}\right\|_{2}$ \\
\hline 0 & $0.101 \mathrm{D}+04$ \\
1 & $0.346 \mathrm{D}+03$ \\
2 & $0.924 \mathrm{D}+01$ \\
3 & $0.130 \mathrm{D}+00$ \\
4 & $0.524 \mathrm{D}-04$ \\
5 & $0.273 \mathrm{D}-09$
\end{tabular}

Boundary condition (5.2)

\begin{tabular}{c|c}
$\nu$ & $\left\|\Delta z_{\nu}\right\|_{2}$ \\
\hline 0 & $0.542 \mathrm{D}+02$ \\
1 & $0.403 \mathrm{D}+02$ \\
2 & $0.208 \mathrm{D}+02$ \\
3 & $0.183 \mathrm{D}+01$ \\
4 & $0.341 \mathrm{D}-02$ \\
5 & $0.120 \mathrm{D}-08$
\end{tabular}

and to add a further boundary condition due to the introduced additional dynamics. Here we can solve this problem in its original formulation. Instead of (5.1), we also used the boundary conditions

$$
v_{2}(0)=v_{2}(2.5)=0
$$

thus seeking a periodic orbit. Observe that we must fix the phase of the solution since the problem is autonomous.

Starting in both cases with the initial guess

$$
\begin{array}{ll}
x_{0}=(1,0.3,0,0,1), & \dot{x}_{0}=(0,0,0,-g, 0), \\
\ddot{x}_{0}=(0,-g, 0,0,0), & x_{0}^{(3)}=(0,0,0,0,0)
\end{array}
$$

and using $N=1$, we obtained solutions according to Table 3 .

Example 5.4 In [19], the model of a (two-dimensional) truck is given. It has the form of a standard multibody system

$$
\begin{aligned}
& \dot{p}=v, \\
& M \dot{v}=f(p, v, u, \dot{u})-g_{p}(p)^{T} \lambda, \\
& g(p)=0,
\end{aligned}
$$

where $p$ are the (generalized) positions, $v$ the corresponding velocities and $\lambda$ the forces introduced by the constraint $g(p)=0$. In the truck model, $p$ and $v$ have eleven components and $\lambda$ is scalar. Hypothesis 2.1 is fulfilled with $\mu=2, d=20$, and $a=3$. The (scalar) function $u$ models the road profile and is chosen here to be

$$
u(t)=\tau \sin (20 \pi t) .
$$

Asking as in [20] for the periodic response of the system for $\tau=0.05$, we require the boundary conditions

$$
\begin{aligned}
& p_{l}(0)=p_{l}(0.1), \quad l=1, \ldots, 9,11, \\
& v_{l}(0)=v_{l}(0.1), \quad l=1, \ldots, 9,11 .
\end{aligned}
$$

This problem suffers from an extremely bad scaling and high nonlinearity. Therefore, we applied a (fixed) scaling to get reasonable condition numbers and used classical homotopy according to

$$
\tau \in\{0.01,0.02,0.03,0.04,0.05\}
$$

to get the desired solution. The homotopy was started with the equilibrium state for $\tau=0$. The course of the Gauß-Newton-like procedure for $N=2$ can be found in Table 4. 
Table 4: Values $\left\|\Delta z_{\nu}\right\|_{2}$ for the homotopy of Example 5.4

\begin{tabular}{c|c|c|c|c|c}
$\nu$ & $\tau=0.01$ & $\tau=0.02$ & $\tau=0.03$ & $\tau=0.04$ & $\tau=0.05$ \\
\hline 0 & $0.440 \mathrm{D}+04$ & $0.481 \mathrm{D}+04$ & $0.523 \mathrm{D}+04$ & $0.527 \mathrm{D}+04$ & $0.464 \mathrm{D}+04$ \\
1 & $0.639 \mathrm{D}+03$ & $0.610 \mathrm{D}+03$ & $0.610 \mathrm{D}+03$ & $0.762 \mathrm{D}+03$ & $0.949 \mathrm{D}+03$ \\
2 & $0.370 \mathrm{D}+02$ & $0.442 \mathrm{D}+02$ & $0.354 \mathrm{D}+02$ & $0.215 \mathrm{D}+02$ & $0.132 \mathrm{D}+02$ \\
3 & $0.110 \mathrm{D}-01$ & $0.359 \mathrm{D}-01$ & $0.825 \mathrm{D}-01$ & $0.614 \mathrm{D}-01$ & $0.288 \mathrm{D}-01$ \\
4 & $0.984 \mathrm{D}-05$ & $0.881 \mathrm{D}-07$ & $0.421 \mathrm{D}-05$ & $0.119 \mathrm{D}-04$ & $0.140 \mathrm{D}-04$ \\
5 & - & - & - & $0.635 \mathrm{D}-08$ & $0.133 \mathrm{D}-07$
\end{tabular}

In summary, we have demonstrated that the presented multiple shooting method is able to solve problems with different values of the index and different structures. The tables show that the developed Gauß-Newton-like method has very good convergence properties. Indeed, for the presented examples they cannot be distinguished from quadratic convergence.

\section{Conclusions}

We have presented a multiple shooting approach for the solution of nonlinear boundary value problems for differential-algebraic systems of arbitrary index and without special structure requirements. Using a specific Gauß-Newton-like method for the solution of the nonlinear system of boundary and continuity conditions we have proved superlinear convergence of the method. We have implemented the method on the basis of a new general solver for DAEs of arbitrary index [13] and demonstrated the numerical properties of the method for several examples.

\section{References}

[1] U. M. Ascher, R. Mattheij, and R. Russell. Numerical Solution of Boundary Value Problems for Ordinary Differential Equations. SIAM, Philadelphia, 2nd edition, 1995.

[2] S. L. Campbell. A general form for solvable linear time varying singular systems of differential equations. SIAM J. Math. Anal., 18:1101-1115, 1987.

[3] S. L. Campbell and E. Griepentrog. Solvability of general differential algebraic equations. SIAM J. Sci. Comput., 16:257-270, 1995.

[4] P. Deuflhard and G. Heindl. Affine invariant convergence theorems for Newton's method and extensions to related methods. SIAM J. Numer. Anal., 16:1-10, 1979.

[5] R. England, R. Lamour, and J. Lopez-Estrada. Multiple Shooting using a Dichotomically Stable Integrator for Solving DAEs. To appear in Appl. Num. Math. 2002.

[6] G. H. Golub and C. F. Van Loan. Matrix Computations. 2nd Ed. The Johns Hopkins University Press, Baltimore, Md, 1996.

[7] E. Hairer and G. Wanner. Solving Ordinary Differential Equations II. Springer-Verlag, Berlin, 1991. 
[8] P. Kunkel and V. Mehrmann. Canonical forms for linear differential-algebraic equations with variable coefficients. J. Comput. Appl. Math., 56:225-251, 1994.

[9] P. Kunkel and V. Mehrmann. A new class of discretization methods for the solution of linear differential-algebraic equations. SIAM J. Numer. Anal., 33:1941-1961, 1996.

[10] P. Kunkel and V. Mehrmann. Regular solutions of nonlinear differential-algebraic equations and their numerical determination. Numer. Math., 79:581-600, 1998.

[11] P. Kunkel and V. Mehrmann. Analysis of over- and underdetermined nonlinear differential-algebraic systems with application to nonlinear control problems. Math. Contr. Sign. Syst., 14:233-256, 2001.

[12] P. Kunkel, V. Mehrmann, W. Rath, and J. Weickert. GELDA: A Software Package for the Solution of General Linear Differential Algebraic equations. SIAM J. Sci. Comp. 18:115-138, 1997.

[13] P. Kunkel, V. Mehrmann, and I. Seufer. GENDA: A software package for the solution of GEneral Nonlinear Differential-Algebraic equations. Technical Report 730-02, Institut für Mathematik, TU Berlin, D-10623 Berlin, FRG, 2002.

[14] P. Kunkel and R. Stöver. Symmetric collocation methods for linear differential-algebraic boundary value problems. Numer. Math., 91:475-501, 2002.

[15] R. Lamour. A Shooting Method for Fully Implicit Index-2 DAEs. SIAM J. Sci. Comput., 18:94-114, 1997.

[16] J. Ortega and W. C. Rheinboldt. Iterative Solutions of Nonlinear Equations in Several Variables. Academic Press, New York, 1970.

[17] T. Schmidt and M. Hou. Rollringgetriebe. Internal Report, Sicherheitstechnische Regelungs- und Meßtechnik, Bergische Universiät, GH Wuppertal, Wuppertal, FRG, 1992.

[18] V. H. Schulz, H. G. Bock, and M. C. Steinbach. Exploiting invariants in the numerical solution of multipoint boundary value problems for DAE. SIAM J. Sci. Comput., 19:440467, 1998.

[19] B. Simeon, F. Grupp, C. Führer, and P. Rentrop. A nonlinear truck model and its treatment as a multibody system. J. Comput. Appl. Math., 50:523-532, 1994.

[20] R. Stöver. Numerische Lösung von linearen differential-algebraischen Randwertproblemen. Dissertationsschrift, Universität Bremen, Bremen, FRG, 1999.

[21] R. Stöver. Collocation methods for solving linear differential-algebraic boundary value problems. Numer. Math., 88:771-795, 2001. 


\section{A Proof of Remark 3.2}

In the linear case, the derivative array equations (2.1) have the form

$$
M_{\ell}(t)\left[\begin{array}{c}
\dot{x} \\
\vdots \\
x^{\ell+1}
\end{array}\right]=N_{\ell}(t) x+g_{\ell}(t)
$$

with

$$
M_{\ell}:[\underline{t}, \bar{t}] \rightarrow \mathbb{R}^{(\ell+1) n,(\ell+1) n}, \quad N_{\ell}:[\underline{t}, \bar{t}] \rightarrow \mathbb{R}^{(\ell+1) n, n}, \quad g_{\ell}:[\underline{t}, \bar{t}] \rightarrow \mathbb{R}^{(\ell+1) n} .
$$

Defining

$$
\begin{array}{lll}
E_{1}(t)=Z_{1}(t)^{T} M_{0}(t), & A_{1}(t)=Z_{1}(t)^{T} N_{0}(t), \quad f_{1}(t)=Z_{1}(t)^{T} g_{0}(t) \\
& A_{2}(t)=Z_{2}(t)^{T} N_{\mu}(t), \quad f_{2}(t)=Z_{2}(t)^{T} g_{\mu}(t)
\end{array}
$$

with $Z_{1}, Z_{2}$ as in the nonlinear case, the given solution $x^{*}$ satisfies the linear DAE

$$
\left[\begin{array}{c}
E_{1}(t) \\
0
\end{array}\right] \dot{x}=\left[\begin{array}{c}
A_{1}(t) \\
A_{2}(t)
\end{array}\right] x+\left[\begin{array}{l}
f_{1}(t) \\
f_{2}(t)
\end{array}\right]
$$

which has vanishing strangeness-index, see $[8,9]$. Following the theory presented there, there exist pointwise nonsingular (smooth) matrix functions $P, Q:[\underline{t}, \bar{t}] \rightarrow \mathbb{R}^{n, n}$ such that

$$
\begin{aligned}
& P(t)\left[\begin{array}{c}
E_{1}(t) \\
0
\end{array}\right] Q(t)=\left[\begin{array}{ll}
I & 0 \\
0 & 0
\end{array}\right] \\
& P(t)\left[\begin{array}{c}
A_{1}(t) \\
A_{2}(t)
\end{array}\right] Q(t)-P(t)\left[\begin{array}{c}
E_{1}(t) \\
0
\end{array}\right] \dot{Q}(t)=\left[\begin{array}{ll}
0 & 0 \\
0 & I
\end{array}\right]
\end{aligned}
$$

transforming the DAE to

$$
\left[\begin{array}{ll}
I & 0 \\
0 & 0
\end{array}\right] \dot{\tilde{x}}=\left[\begin{array}{ll}
0 & 0 \\
0 & I
\end{array}\right] \tilde{x}+\left[\begin{array}{c}
\tilde{f}_{1}(t) \\
\tilde{f}_{2}(t)
\end{array}\right]
$$

with

$$
x(t)=Q(t) \tilde{x}(t)=Q(t)\left[\begin{array}{c}
\tilde{x}_{1}(t) \\
\tilde{x}_{2}(t)
\end{array}\right], \quad\left[\begin{array}{l}
\tilde{f}_{1}(t) \\
\tilde{f}_{2}(t)
\end{array}\right]=P(t)\left[\begin{array}{c}
f_{1}(t) \\
f_{2}(t)
\end{array}\right] .
$$

Hence, all solutions have the form

$$
x(t)=Q(t)\left[\begin{array}{c}
\tilde{x}_{1}(\underline{t})+\int_{\underline{t}}^{t} \tilde{f}_{1}(s) d s \\
-\tilde{f}_{2}(t)
\end{array}\right]
$$

implying that

$$
\Phi_{x, y}(x, y)=Q(\bar{t})\left[\begin{array}{ll}
I & 0 \\
0 & 0
\end{array}\right]\left[Q(\underline{t})^{-1} \quad 0\right] .
$$

The equations (3.1) that define $S$ take the form

$$
M_{\mu}(\underline{t}) \hat{y}-N_{\mu}(\underline{t}) \hat{x}-g_{\mu}(\underline{t})=0, \quad T_{2}(\underline{t})^{T}(\hat{x}-x)=0, \quad T_{1}(\underline{t})^{T}(\hat{y}-y)=0 .
$$


Multiplying the first relation with $Z_{2}(\underline{t})^{T}$, we obtain

$$
Z_{2}(\underline{t})^{T} N_{\mu}(\underline{t}) \hat{x}=-Z_{2}(\underline{t})^{T} g_{\mu}(\underline{t})
$$

and therefore (recalling the definition of $A_{2}$ and $f_{2}$ )

$$
\hat{x}=\left[\begin{array}{c}
A_{2}(\underline{t}) \\
T_{2}(\underline{t})^{T}
\end{array}\right]^{-1}\left[\begin{array}{c}
-f_{2}(\underline{t}) \\
T_{2}(\underline{t})^{T} x
\end{array}\right] .
$$

A similar argument yields

$$
\hat{y}=\left[\begin{array}{c}
Z_{2}^{\prime}(\underline{t})^{T} M_{\mu}(\underline{t}) \\
T_{1}(\underline{t})^{T}
\end{array}\right]^{-1}\left[\begin{array}{c}
Z_{2}^{\prime}(\underline{t})^{T} N_{\mu}(\underline{t}) \hat{x}+Z_{2}^{\prime}(\underline{t})^{T} g_{\mu}(\underline{t}) \\
T_{1}(\underline{t})^{T} y
\end{array}\right] .
$$

Altogether, we have

$$
\Phi_{x, y}(x, y) S_{x}(x, y)=Q(\bar{t})\left[\begin{array}{ll}
I & 0 \\
0 & 0
\end{array}\right] Q(\underline{t})^{-1}\left[\begin{array}{c}
A_{2}(\underline{t}) \\
T_{2}(\underline{t})^{T}
\end{array}\right]^{-1}\left[\begin{array}{c}
0 \\
T_{2}(\underline{t})^{T}
\end{array}\right]
$$

and therefore

$$
\mathcal{E}=C T_{2}(\underline{t})+D Q(\bar{t})\left[\begin{array}{ll}
I & 0 \\
0 & 0
\end{array}\right] Q(\underline{t})^{-1}\left[\begin{array}{c}
A_{2}(\underline{t}) \\
T_{2}(\underline{t})^{T}
\end{array}\right]^{-1}\left[\begin{array}{l}
0 \\
I
\end{array}\right] .
$$

Following [20], the transformation $P$ has the block structure

$$
P(t)=\left[\begin{array}{cc}
P_{11}(t) & P_{12}(t) \\
0 & P_{22}(t)
\end{array}\right]
$$

where $P_{22}:[\underline{t}, \bar{t}] \rightarrow \mathbb{R}^{a, a}$ is pointwise nonsingular. Hence,

$$
P_{22}(\underline{t}) A_{2}(\underline{t}) Q(\underline{t})=\left[\begin{array}{ll}
0 & I
\end{array}\right]
$$

and $Q(\underline{t})$ has the form

$$
Q(\underline{t})=\left[T_{2}(\underline{t}) U *\right]
$$

with some nonsingular matrix $U \in \mathbb{R}^{d, d}$. In particular,

$$
Q(\underline{t})\left[\begin{array}{c}
U^{-1} \\
0
\end{array}\right]=T_{2}(\underline{t}) .
$$

Defining $\left[C_{11} C_{12}\right]=C Q(\underline{t}),\left[\begin{array}{ll}D_{11} & D_{12}\end{array}\right]=D Q(\bar{t})$, and using

$$
\left[\begin{array}{c}
A_{2}(\underline{t}) \\
T_{2}(\underline{t})^{T}
\end{array}\right] T_{2}(\underline{t})=\left[\begin{array}{l}
0 \\
I
\end{array}\right]
$$

we find

$$
\begin{aligned}
\mathcal{E} & =C Q(\underline{t}) Q(\underline{t})^{-1} T_{2}(\underline{t})+D Q(\bar{t})\left[\begin{array}{cc}
I & 0 \\
0 & 0
\end{array}\right] Q(\underline{t})^{-1} T_{2}(\underline{t})= \\
& =\left[\begin{array}{ll}
C_{11} & C_{22}
\end{array}\right]\left[\begin{array}{c}
U^{-1} \\
0
\end{array}\right]+\left[\begin{array}{ll}
D_{11} & D_{22}
\end{array}\right]\left[\begin{array}{ll}
I & 0 \\
0 & 0
\end{array}\right]\left[\begin{array}{c}
U^{-1} \\
0
\end{array}\right]= \\
& =\left(C_{11}+D_{11}\right) U^{-1}
\end{aligned}
$$

Thus, $\mathcal{E}$ is nonsingular if and only if $C_{11}+D_{11}$ is nonsingular. 